\title{
ANALYSIS OF A NUCLEAR ENHANCED AIRBREATHING ROCKET FOR EARTH TO ORBIT APPLICATIONS
}

\author{
Robert B. Adams* \\ National Aeronautics and Space Administration \\ Marshall Space Flight Center \\ MSFC, AL 35812 \\ D. Brian Landrum ${ }^{\dagger}$ \\ University of Alabama in Huntsville \\ Propulsion Research Center \\ Tech Hall, 5234 \\ Huntsville, Alabama 35899
}

The proposed engine concept is the Nuclear Enhanced Airbreathing Rocket (NEAR). The NEAR concept uses a fission reactor to thermally heat a propellant in a rocket plenum. The rocket is shrouded, thus the exhaust mixes with ingested air to provide additional thermal energy through combustion. The combusted flow is then expanded through a nozzle to provide thrust.

\section{Fission Power Systems}

Nuclear reactions can be divided into two categories: fission and fusion reactions. Significant work has been performed in fission reactor design for space flight applications. In the 1960's the Nuclear Engine for Rocket Vehicle Application (NERVA) project developed a nuclear thermal fission reactor designed for launch applications ${ }^{1}$. This project reached the ground testing stage before it was canceled. The more recently developed SP-100 and SPAR reactors are designed to provide power for electric based engines and onboard subsystems ${ }^{2}$.

The NERVA reactor concept is used in this study as the power source for the NEAR concept. A schematic of a NERVA reactor is shown in Figure 1. The NERVA reactor was chosen for its relatively mature technology. Calculations based on the NERVA concept yield conservative performance for a nuclear reactor in a launch scenario. A particle bed reactor design yields power to weight ratios nearly an order of magnitude greater than NERVA. Nuclear testing of single fuel elements and criticality experiments of a prototype core was achieved before the particle bed reactor program was discontinued.

The NEAR computer code is designed to model the flow through a shrouded rocket where the rocket is powered by nuclear heating of the propellant. Figure 2 illustrates the geometry of the NEAR engine. The code calculates flow properties at each individual station shown in the figure by applying the appropriate closed form compressible and conservation 
relations. The code also sizes the reactor based on user input. Finally performance values for the engine are calculated. Each of these functions is described in further detail below.

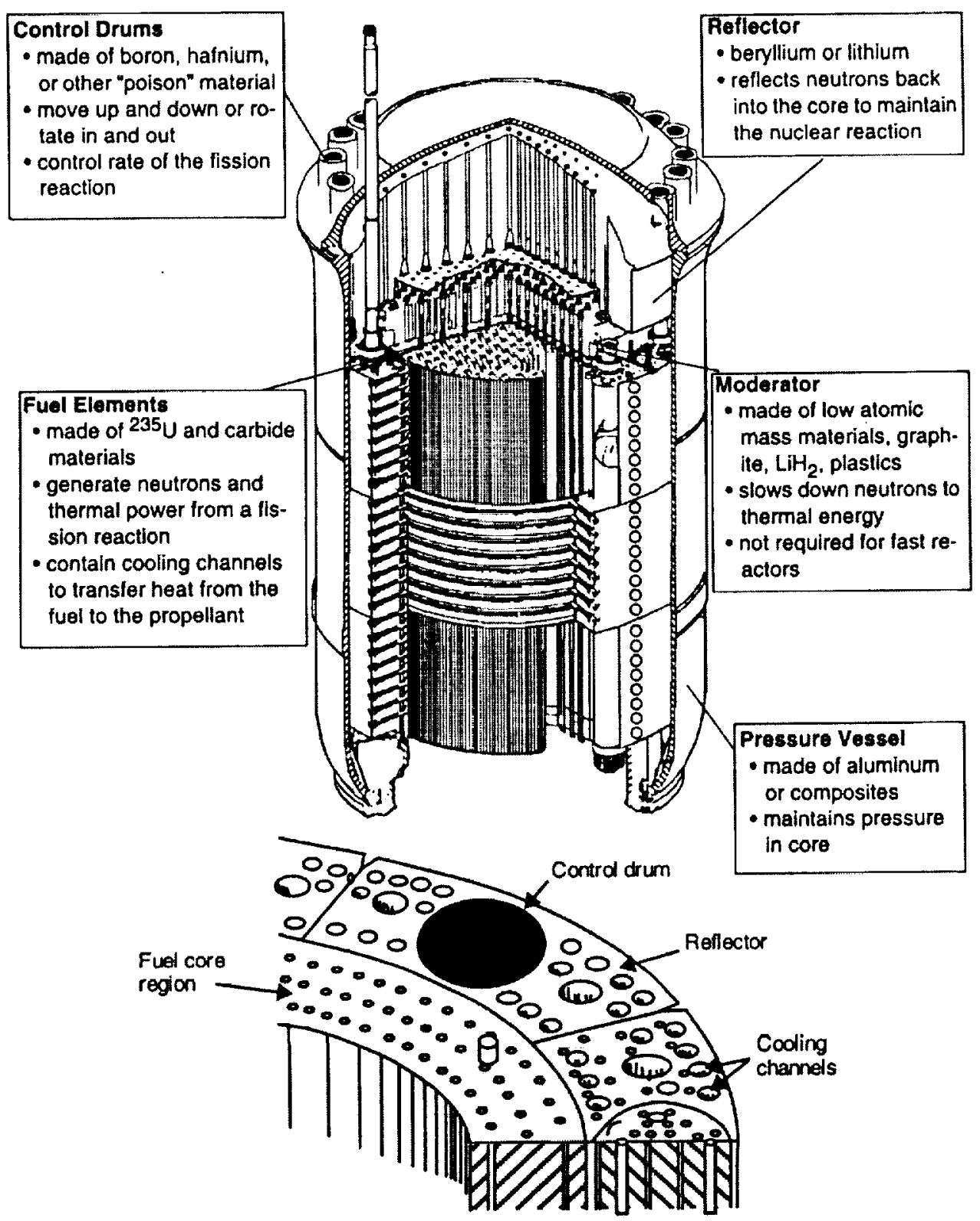

Figure 1 Schematic of a NERVA style fission reactor ${ }^{3}$ 


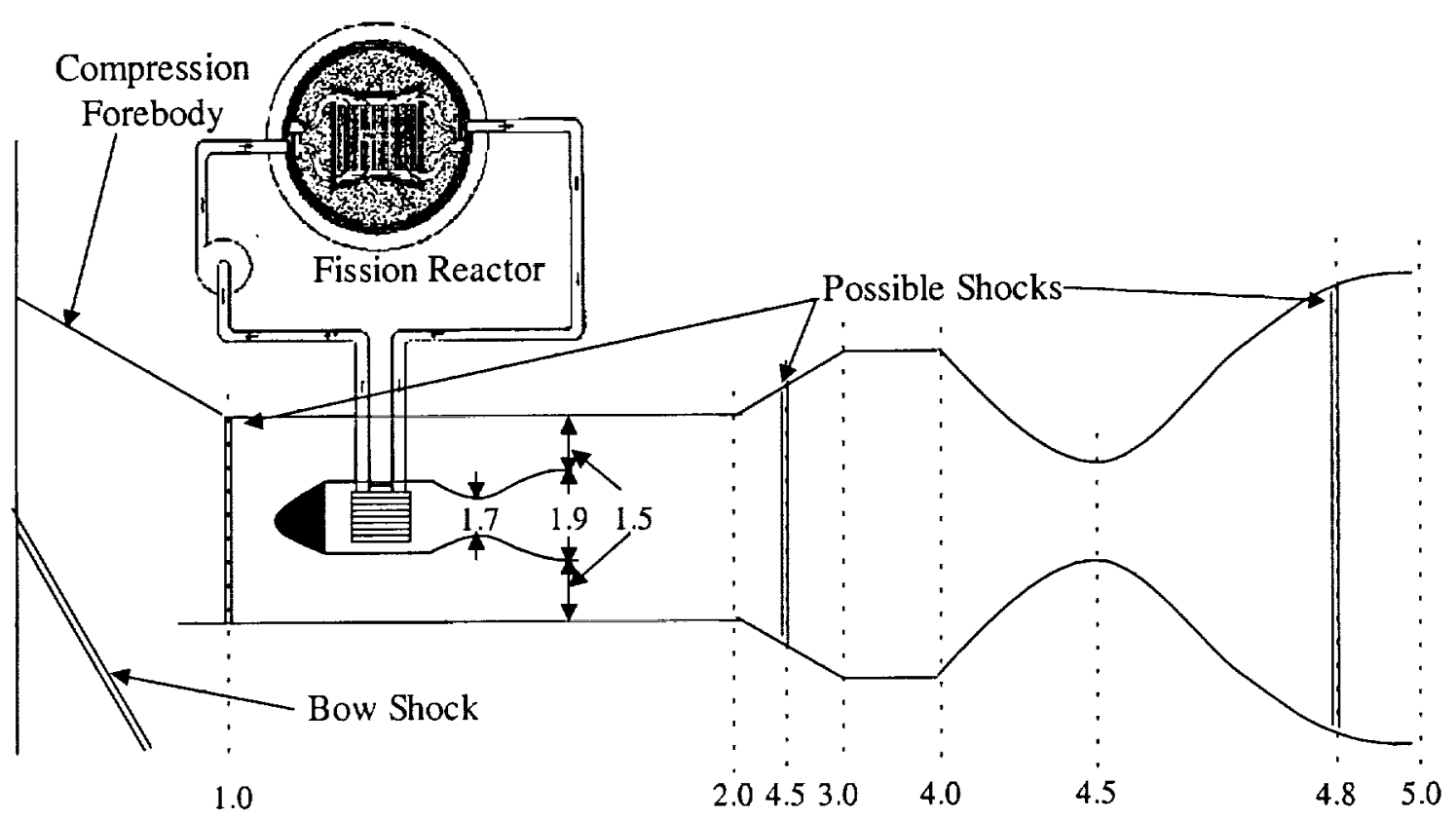

Figure 2 Schematic of NEAR engine

\section{Atmospheric and Trajectory Model}

The NEAR code does not currently incorporate an atmospheric model. Atmospheric data must be determined externally. This study uses the 1976 U.S. Standard Atmosphere as defined in the software included in Heiser and Pratt. The actual atmospheric conditions the vehicle experiences are a function of the vehicle trajectory. The flight trajectory and consequent atmospheric conditions used in this study will be discussed in the results chapter.

\section{Compression Inlet Model}

The initial EPSURBCC code assumed a 'pod' formation, i.e. the engine performance was not affected by the flowfield around the vehicle. The NEAR code incorporates external flowfield effects by assuming that the vehicle is designed with a compression forebody.

As will be seen, the NEAR code calculates the amount of external air that is entrained into the engine flow through the pumping effect of the rocket exhaust. The upstream shock configuration will be affected by this pumping effect. When a compression forebody or inlet experiences a shock structure, which completely covers the engine inlet (such as a normal shock in the isolator or a bow shock that impinges on the cowl lip) there is a specific mass flow rate associated with the shock structure. Thus the upstream shock structure becomes a function of the entrained air. Such conditions will be experienced when the vehicle flies at higher freestream 
Mach numbers. A pumping effect that entrains more air than would normally be captured from the compression forebody suggests that the upstream bow shock would curve towards the cowl and that the isolator normal shock would be 'swallowed' by the engine. A pumping effect that entrains less air would have the opposite effect. Determination of the upstream shock configuration under such conditions requires complex iteration procedure between the forebody and pumping engine that has not been integrated into the code. To avoid this problem the code assumes that there is a varying area immediately after the compression forebody. The varying area allows placement of the upstream shocks dictated by the entrained air mass flow rate.

\section{Engine Model}

The NEAR code operates by calculating gasdynamic properties at several stations through the engine flowfield. The stations are indicated numerically, as can be seen in Figure . The stations are strategically located so that the difference between each location can be calculated using closed form compressible relations. In addition the interaction between the rocket and air streams is modeled by a set of quasi-empirical relations for mixing and combustion of two high-speed streams.

\section{Description of NEAR stations}

Each of the stations used in the NEAR code are described below. The rocket exhaust is described as the primary airstream. In the same manner, the entrained airstream is commonly referred to as the secondary airstream. Also note that the overall NEAR system is referred to as the engine. Use of the word rocket indicates the rocket section (stations rocket, 1.7 and 1.9) only.

\section{Freestream}

Not shown in Figure 2, the freestream properties are given in the user input. Such conditions are typically found through the combined use of an assumed trajectory and an atmospheric database.

\section{Station 0.5}

This is the flow condition at the engine inlet. These values are determined through use of a compression forebody program. For the purposes of this project the HyFIM model is used.

\section{Station 1.0}

The NEAR code assumes subsonic flow for the entrained air. Towards this end the engine isolator is modeled by a single normal shock should the properties at station 0.5 be 
supersonic. The conditions at station 1.0 are then determined using normal shock relations found in any compressible text.

\section{Station 1.5}

Station 1.5 represents the flow properties of the secondary stream at the point of minimum area. As can be seen in Figure 2 station 1.5 coincides with the plane defined by the outer edge of the rocket nozzle.

Rocket

Rocket indicates the rocket plenum only. Stations 1.7 and 1.9 complete the stations used to represent the flow through the rocket portion

\section{Station 1.7}

Station 1.7 defines the rocket throat position. The code assumes that the rocket exhaust will be supersonic and insures that the primary flow can expand supersonically against the secondary flow backpressure.

\section{Station 1.9}

Station 1.9 represents the primary flow conditions at the rocket exhaust point. Note that the area at station 1.5 and 1.9 does not necessarily equal that at station 2.0. The rocket nozzle will have a finite thickness and there may be other surrounding structure that would represent the difference in areas.

Station 2.0

The mixed and combusted products of the primary and secondary streams are represented in Station 2.0. A mixing and combustion model determines the amount of each stream that is actually combusted. The average properties of the three streams (unmixed air, unmixed fuel, and combustion products) are assigned to the properties at station 2.0.

\section{Station 2.5}

If the flow at station 2.0 is supersonic a shock exists somewhere between stations 2.0 and 3.0. The shock is assigned station 2.5 .

\section{Station 3.0}

Station 3.0 represents an area change inside the engine from station 2.0. The properties at station 3.0 are determined from station 2.0 using quasi-one-dimensional equations.

\section{Station 4.0}

Station 4.0 represents the start of a converging-diverging nozzle. The initial EPSURBCC program allowed for a secondary $\mathrm{H} 2$ injection between stations 3.0 and 4.0. The properties at station 4.0 were calculated using Reynolds flow equations for compressible constant area flow 
with heat release. The secondary injection was removed from the NEAR code and may be replaced at a later date with a new injection system that incorporates a mixing model. At the present the conditions at station 4.0 are the same as those at station 3.0.

Station 4.5

Station 4.5 represents the throat of a converging-diverging nozzle. The properties here are determined from isentropic flow relations.

\section{Station 4.8}

If the throat at station 4.5 is sonic and the freestream pressure is high enough the nozzle may experience a shock inside the diverging section. This shock is assigned the station 4.8 and the properties before and after the shock are determined using normal shock relations.

\section{Station 5.0}

Station 5.0 represents the exhaust plane of the NEAR engine. The conditions here are calculated using isentropic flow relations.

\section{Mixing and Combustion Model}

The EPSURBCC code assumed that the engine flow achieved fully mixed equilibrium conditions. This assumption is optimistic for the high-speed flows experienced in combined cycle engines. A mixing and combustion model was incorporated in the model to give a more accurate representation of the actual engine flow. The model, described below, is described in more detail in reference 4 .

Mixing and combustion of two miscible streams can be described as the confluence of three separate processes. The two streams are typically at different velocities, therefore, there will be a viscous interaction between the two layers. The mixing of the two streams will obviously occur within the shear layer, as the flow outside the shear layer is by definition undisturbed. The entire viscous layer will not become fully mixed in the mixing lengths of interest for a combined cycle engine, thus only a fraction of the viscous layer is fully mixed. A portion of the mixed layer will combust and generate products, which is represented by another fraction. Thus the confluence of three separate processes accounts the amount of combustion products as calculated by the product of three ratios as seen in Equation (1).

$$
\frac{\delta_{p}}{x}=\frac{\delta}{x} \frac{\delta_{m}}{\delta} \frac{\delta_{p}}{\delta_{m}}
$$


The first term represents the viscous interaction between the two flows. For incompressible shear layers the shear layer growth is expected to be a function of the density and velocity ratios of the two streams. Dimotakis proposed the shear layer growth as

$$
\frac{\delta}{x}=C_{\delta} \frac{(1-r)(1+\sqrt{s})}{2(1+r \sqrt{s})} \frac{\frac{1-\sqrt{s}}{1+\sqrt{s}}}{1+2.9 \frac{1+r}{1-r}}
$$

where

$$
r=\frac{u_{2}}{u_{1}}
$$

and

$$
s=\frac{\rho_{2}}{\rho_{1}} .
$$

Subscript 1 represents the high speed or primary flow and subscript 2 represents the lower speed or secondary flow. The parameter $x$ is the axial mixing length of interest. In the equations above the coefficient $C_{\delta}$ is believed to be independent from $r$ and $s$. The range of $C_{\delta}$ has been experimentally determined to be between 0.25 and 0.45 . The dependence of $C_{\delta}$ and a final determination of its possible range have yet to be determined.

High convective Mach numbers in the shear layer will affect the growth rate of the shear layer. The convective Mach number is defined as

$$
M_{c 1}=\frac{u_{1}-u_{c}}{a_{1}}, \quad M_{c 2}=\frac{u_{r}-u_{2}}{a_{2}} .
$$

For subsonic convective Mach numbers it is expected that both flows will experience approximately equal pressure recovery. Thus,

$$
\left[1+\frac{\gamma_{1}-1}{2} M_{c 1}^{2}\right]^{\frac{\gamma_{1}}{\gamma_{1}-1}}=\left[1+\frac{\gamma_{2}-1}{2} M_{c 2}^{2}\right]^{\frac{\gamma_{2}}{\gamma_{2}-1}}
$$

After substituting equation (5) into (6) $u_{c}$ can be determined through iteration, as all of the conditions of the primary and secondary streams are known. Once $u_{c}$ is determined, equation (5) yields the convective Mach numbers. A curvefit of the available experimental data, performed by Dimotakis, yields, 


$$
\frac{\frac{\delta}{x}\left(r, s, M_{c 1}\right)}{\frac{\delta}{x}\left(r, s, M_{c 1}=0\right)}=\left(1-f_{\infty}\right) e^{-3 M_{r 1}^{2}}+f_{\infty},
$$

where $f_{\infty}$ is a curvefit factor with a value of 0.2 . Multiplication of the above with equation (2) yields the shear layer growth for a compressible flow.

Dimotakis also reports values for the fraction of products from the shear layer. These fractions depend on the stochiometric mole fraction of the reactants in question. The data is experimental and unfortunately the mole fraction of gaseous $\mathrm{H} 2$ and air, the constituents at interest here, are not reported. The code currently uses the lowest ratio reported, 0.125 , which corresponds to a mole fraction much lower than that of $\mathrm{H} 2$ and air. The data suggests that higher mole fractions yield higher combustion ratios, thus the value of 0.125 is deemed to be conservative. This factor is multiplied with the product of equations (2) and (7) to yield the final ratio of the product delta with axial mixing length.

The shear layer will propagate into each stream asymmetrically. Typically the shear layer will propagate into the high stream flow faster than into the low stream flow. The ratio of the primary flow delta to the secondary flow delta is known as the entrainment ratio and is defined as

$$
E_{v}=\frac{u_{1}-u_{c}}{u_{c}-u_{2}}\left(1+C_{l} \frac{1-r}{1+r}\right) \text {. }
$$

where $C_{1}$ is an experimentally determined coefficient.

The amount of each stream that is converted into combustion products can now be calculated. First the combustion delta, $\delta p$, is determined from the relations above. The primary and secondary delta is calculated from the equivalence ratio above. A portion of both the secondary and primary stream is captured in the combustion shear layer. The areas of the captured portions of those streams are determined from the primary and secondary delta respectively. Finally the product of the relative area, density and velocity pertaining to the captured primary and secondary streams defines the mass flow rates of those streams.

It should be noted that Dimotakis determined the relations above for two infinite parallel streams. In our application the actual flow is a circular primary stream bordered by a rectangular secondary stream. The implied polar geometry changes the propagation of the delta functions into each flow. This effect was considered to be of lower order and was not included in the mixing and combustion calculations. 


\section{Reactor Model}

The NEAR code incorporates a model of a NERVA design nuclear reactor with shielding. The reactor size and mass is based on several assumptions relevant to the design and the defined hydrogen mass flow and exit temperature. The analysis used to model the reactor is recounted below and is based on chapter 8 of Humble, Henry and Larson ${ }^{3}$.

The power produced by the reactor is a function of the mass flow rate and exit temperature of the propellant. Hydrogen is assumed to be the propellant of choice for this application. Since the initial hydrogen input is in liquid state the power requirement must include the heat of vaporization. The heat of vaporization is a function of the system pressure. For the purposes of this analysis the variance in the heat of vaporization can be neglected, which allows for a simpler model of the power-exit temperature relationship. The power-exit temperature relationship is defined by

$$
P=\dot{m}\left(0.018061 T_{2}-5.715417\right) \text {. }
$$

where $P$ is power in MW, $T_{2}$ is the exit temperature in $\mathrm{K}$ and $\dot{m}$ is the mass flow rate in $\mathrm{kg} / \mathrm{sec}$.

The core volume is estimated next. The core requires a certain minimum volume to produce the required power. The relationship between volume and power is dictated by the power density, which has an approximate value of $1570 \mathrm{MW} / \mathrm{m}^{3}$ for NERVA reactors. As the reactor operates some of the core uranium will fission. The core must accommodate the loss of the 'burned' uranium if the reactor is to operate at the required power level for a sustained period. The relationship between the power level and the core volume is dictated by

$$
V=P\left(6.4 \cdot 10^{-13} t_{b}+\frac{1}{P_{D}}\right) \text {. }
$$

Where $P_{D}$ is the aforementioned power density, $P$ is the power in $\mathrm{MW}, V$ is the volume in $\mathrm{m}^{3}$, and $t_{b}$ is the burn duration (or reactor operating time) in seconds.

Shielding calculations require determination of the reactor radius and height. The reactor is modeled as a right circular cylinder. The volume equation for such a geometric shape is

$$
V=\pi R^{2} H
$$

Since there are two unknowns another equation must be found. Lawrence et al. ${ }^{3}$ defined the material buckling parameter as

$$
B_{m}=\sqrt{\frac{1-P_{n l}}{P_{n l}\left(L^{2}+\tau\right)}} .
$$


where $\tau$ represents the average lifetime of the neutrons in the core and $L$ refers to the thermal diffusion length in the core..

$P_{n l}$ is the nonleakage probability, or the fraction of the neutrons that remain in the core.

It is defined as

$$
P_{n l}=\frac{k_{e f f}}{p \eta \varepsilon f} .
$$

Where $k_{e f f}$ is the criticality factor, $p$ is the resonance escape probability, $f$ is the thermal utilization factor, $\varepsilon$ is the fast fission factor, and $\eta$ is the neutron production effectiveness factor. A further discussion of these parameters can be found in reference 3.

Once the nonleakage probability has been determined equation (12) will yield the buckling parameter. The buckling parameter defines the dimensions of the core through

$$
B_{m}^{2}=B_{G}^{2}=\left(\frac{\pi}{H}\right)^{2}+\left(\frac{2.405}{R}\right)^{2} \text {. }
$$

Concurrent solution of equations (11) and (14) allows determination of the core radius and height.

The overall mass of the reactor can now be determined. A typical core density for a NERVA reactor is $2300 \mathrm{~kg} / \mathrm{m}^{3}$. The density of uranium is $19100 \mathrm{~kg} / \mathrm{m}^{3}$. The difference in densities is the reactor volume devoted to moderators and cooling passages. The product of the core density and core volume yields the core mass. The shielding mass is dependant on the shield configuration. This analysis assumes a shield comprised of $18 \mathrm{~cm}$ of Beryllium $(\mathrm{Be}), 5 \mathrm{~cm}$ of Tungsten (W) and $5 \mathrm{~cm}$ of Lithium Hydride $\left(\mathrm{LiH}_{2}\right)$. Beryllium is placed closest to the reactor because it is an excellent neutron reflector. The very dense Tungsten is next, as it has good neutron and gamma ray absorption properties. The Lithium Hydride is a good compromise between neutron absorption properties and lighter weight material. The combination of the three materials yields a neutron attenuation of 9 orders of magnitude $\left(4 \times 10^{-9}\right)$ and a gamma ray attenuation of 3 orders of magnitude (0.00105). The mass of the shielding is calculated assuming an inner Beryllium radius equal to the core radius. The volumes of the shielding material can then be calculated. Densities of the shielding materials are public knowledge and yield the shielding mass from the volume calculations. 


\section{Trajectory Analysis of Proposed Concept}

\section{Atmosphere and Trajectory}

Determination of an optimized mission performance for a given engine concept would require a trajectory simulation on a code such as POST3d. Such a simulation would require definition of a vehicle geometry and calculations on the lift and drag on the vehicle through the trajectory. Such detail was of secondary priority, due to the time constraints for this study. In lieu of a detailed trajectory study, the authors chose to use the baseline trajectory described by Billig. This trajectory, combined with the 1976 Standard Atmosphere, gives the freestream properties experienced by the engine throughout the mission. Table 1 lists the freestream properties as a function of Mach number for the mission analysis.

Table 1 Trajectory and atmospheric properties for airbreathing range of flight

\begin{tabular}{lccccl}
\hline Mach & $\begin{array}{c}\text { Altitude } \\
(\mathrm{ft})\end{array}$ & $\begin{array}{c}\text { Pressure } \\
\left(\mathrm{lbf} / \mathrm{ft}^{2}\right)\end{array}$ & $\begin{array}{c}\text { Temperature } \\
(\mathrm{R})\end{array}$ & $\begin{array}{c}\text { Density } \\
\left(\mathrm{lbm} / \mathrm{ft}^{3}\right)\end{array}$ & $\begin{array}{c}\text { Speed sound } \\
(\mathrm{ft} / \mathrm{sec})\end{array}$ \\
\hline 2.0 & 36225 & 471.024 & 390.0 & 0.02264 & 968.1 \\
2.5 & 42460 & 349.488 & 390.0 & 0.01679 & 968.1 \\
3.0 & 47951 & 268.704 & 390.0 & 0.01291 & 968.1 \\
3.5 & 52915 & 212.0 & 390.0 & 0.01018 & 968.1 \\
4.0 & 57480 & 170.4 & 390.0 & 0.008187 & 968.1 \\
4.5 & 61731 & 139.0 & 390.0 & 0.066820 & 968.1 \\
5.0 & 65722 & 114.9 & 390.0 & 0.005522 & 968.1 \\
5.5 & 69599 & 95.5 & 392.0 & 0.004567 & 970.6 \\
6.0 & 73300 & 80.2 & 394.0 & 0.003813 & 973.1 \\
6.5 & 76838 & 67.9 & 396.0 & 0.003212 & 975.5 \\
7.0 & 80073 & 58.3 & 397.7 & 0.002748 & 977.7 \\
\hline
\end{tabular}

Inlet

Inlet compression efficiencies are a function of the number of shocks used to achieve the necessary compression. Using more shocks of weaker strength as opposed to one strong shock allows the flow to be turned into the engine section without an excessive increase in static temperature. The inlet used for both engine concepts incorporated four shocks derived from successive 5 degree turning ramps and one cowl shock turning the flow back the full twenty degrees. The lower Mach numbers (2.0 and 2.5) experience detached cowl shocks when the 
relatively low speed flow is forced to turn through the 20-degree angle. The HyFIM code is currently not capable of modeling detached shocks. In these instances it is assumed that the vehicle uses a rotating cowl to eliminate the need to turn the flow at the cowl. The flow then experiences the normal shock used in the NEAR code to reduce the flow to subsonic speeds. The flow is then turned parallel with the engine with no shock.

The NEAR engine operates between Mach 2.0 and Mach 6.0. The NEAR code requires a subsonic entry flow. Decelerating freestream flows of Mach 6.0 and greater to subsonic is prohibitive in terms of temperature rise and entropy loss. The Mach 2.0 condition is selected as a ramjet inlet is usually well started at that freestream condition. The user-defined geometry for this analysis is shown in Table 2. These variables were selected to yield a vehicle geometry similar to that of a shuttle orbiter as was done in chapter 8. A converging-diverging nozzle was used in this performance analysis for a fuller expansion of the exhaust gases. The mixing length used is $35 \mathrm{ft}$., which is similar to the $40 \mathrm{ft}$. length used in the SHX system, but allows five feet for the length of the rocket. The rocket pressure and temperature was selected based on the capabilities of a NERVA reactor ${ }^{3}$. The freestream conditions used in this analysis are those defined in Table 1.

Table 2 Inputs for NEAR trajectory analysis

\begin{tabular}{ll}
\hline \multicolumn{1}{c}{ variable } & \multicolumn{1}{c}{ Value } \\
\hline igeom & 2 \\
area05 & $16792 \mathrm{in}^{2}$ \\
area10 & $5184 \mathrm{in}^{2}$ \\
area15 & $4000 \mathrm{in}^{2}$ \\
area17 & $100 \mathrm{in}^{2}$ \\
area19 & $600 \mathrm{in}^{2}$ \\
area20 & $5184 \mathrm{in}^{2}$ \\
area30 & $10000 \mathrm{in}^{2}$ \\
area40 & $10000 \mathrm{in}^{2}$ \\
area45 & $4000 \mathrm{in}^{2}$ \\
area50 & $62208 \mathrm{in}^{2}$ \\
xmix & $35 \mathrm{ft}$ \\
rockpo & $1000 \mathrm{psi}^{2}$ \\
rockto & $4140 \mathrm{R}$ \\
\hline
\end{tabular}


The mass flow rate of the rocket $\mathrm{H}_{2}$ was varied to calculate performance for several air/fuel ratios for a given Mach number. In this way the peak performance for the given vehicle geometry was captured. Figure 3 illustrates the variance in specific impulse with air/fuel ratio for the Mach numbers in question. Figure 4 is a similar comparison of thrust vs. air/fuel ratio. The stochiometric air/fuel ratio is also indicated on this graph. Several trends can be seen here. First the maximum specific impulse exhibits a upward trend from Mach 2.0 to Mach 4.0 and then back down from Mach 4.5 to Mach 6.0. The engine is undergoing two separate processes that produce thrust. The first is the power delivered by the reactor in the form of heated propellant. The second is the secondary combustion of the air and propellant. The thermal energy from both of these reactions is converted to kinetic energy in the converging-diverging nozzle. The nozzle can only convert so much energy and the amount of conversion is a function of the freestream properties. Any more energy delivered (in the form of more fuel run through the reactor) is wasted. A less than optimal fuel injection rate causes the engine to ingest a superfluous amount of air and its associated drag. Apparently, for this geometry, the balance between area capture and expansion is best around Mach 4.0.

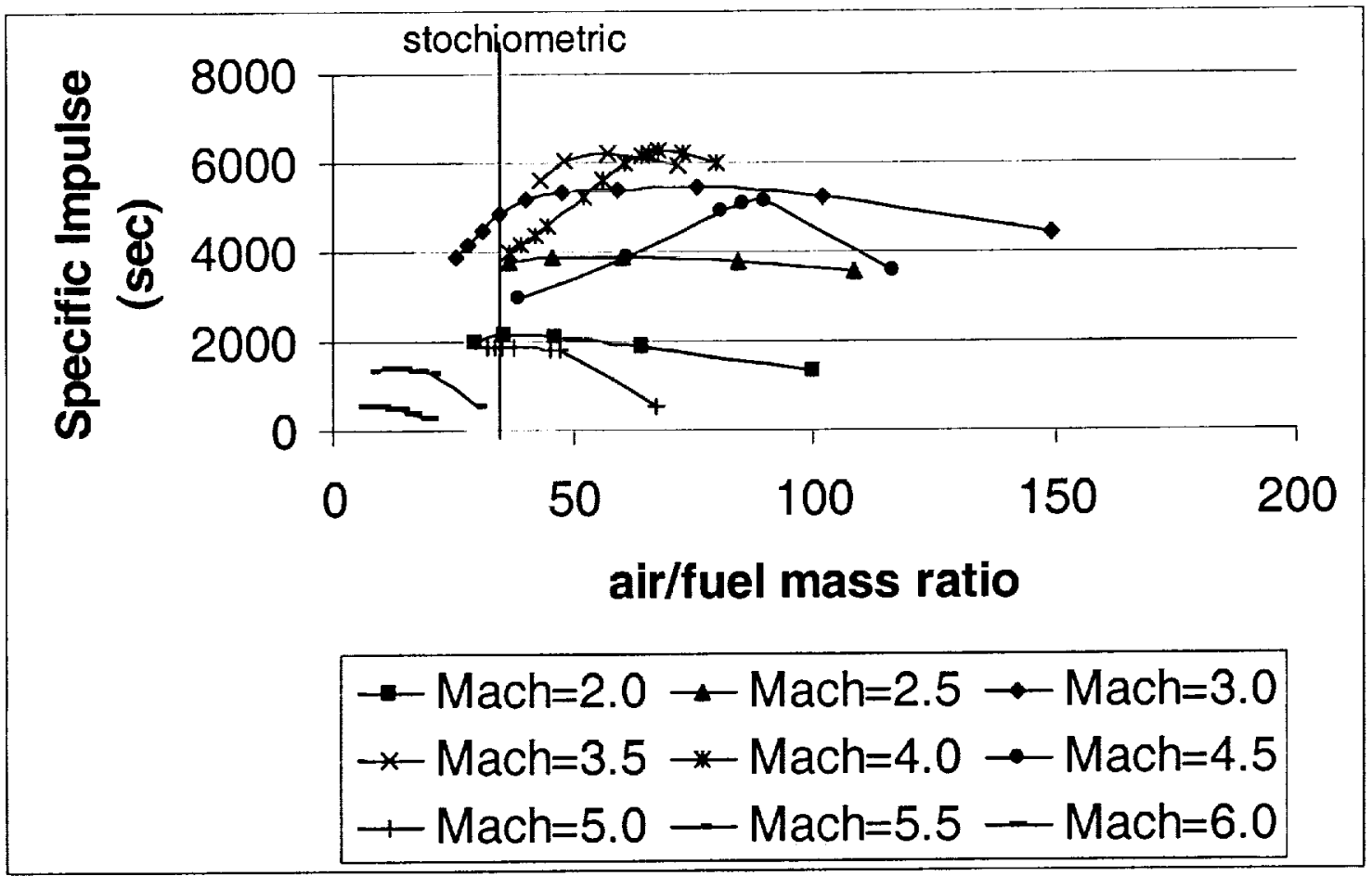

Figure 3 NEAR Specific Impulse vs. A/F ratio at progressive freestream Mach numbers 


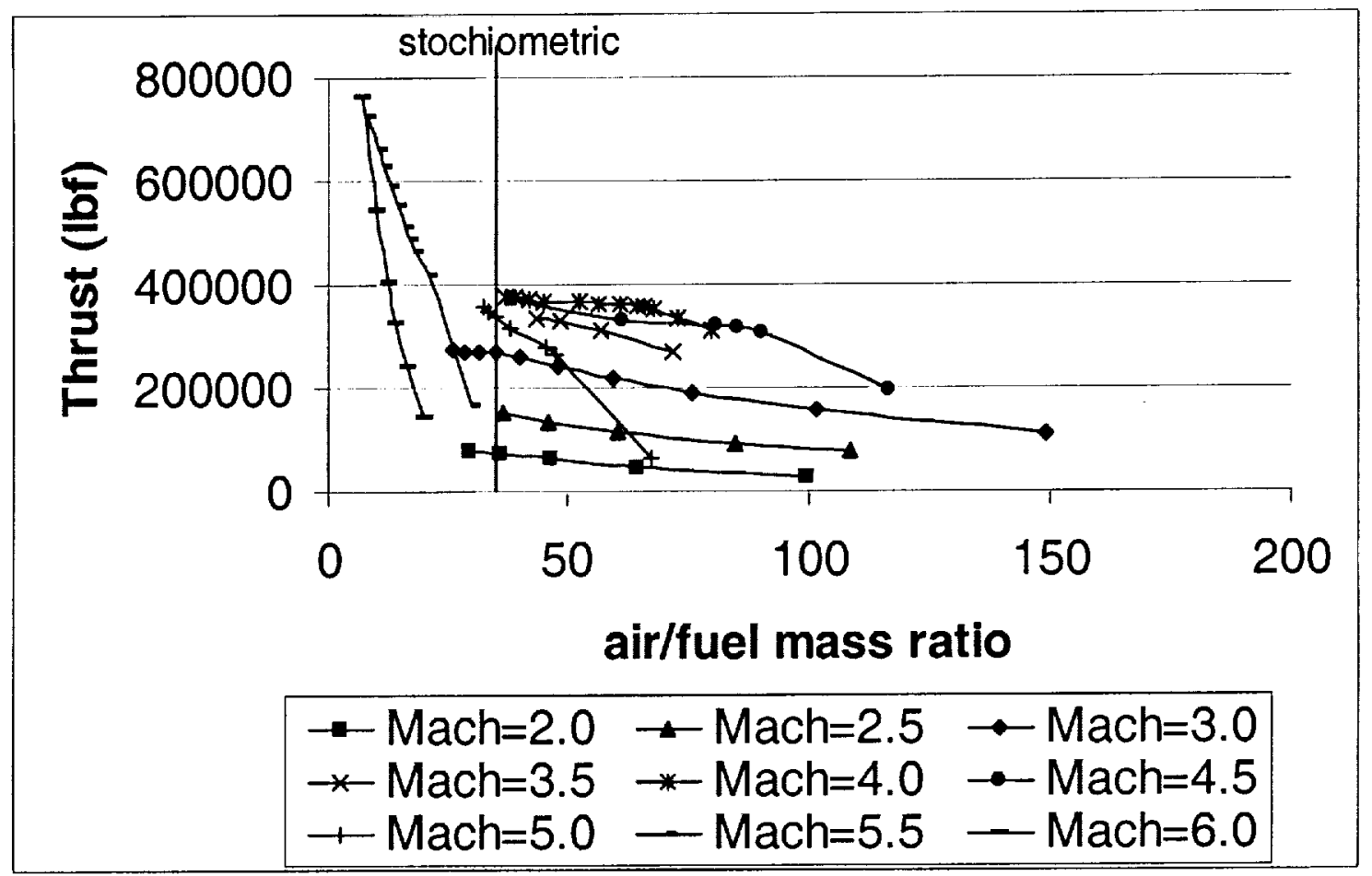

Figure 4 NEAR Thrust vs. A/F ratio at progressive freestream Mach numbers

Lean mixtures yield the peak performance for the lower Mach numbers. In fact the peak efficiency is just right of the stochiometric line for Mach 2.0. The peak operating point grows progressively leaner all the way to Mach 4.5. Note that the engine is swallowing more air as the Mach number increases. Higher Mach numbers incidate higher air intake, which suggests higher fuel usage. The engine nozzle cannot hope to convert the energy input from combustion of a stochiometric mixture; thus the mixture is progressively leaned to operate the nozzle at peak performance.

The fuel rich mixtures for Mach numbers of 5.0 and above indicate that the thrust from the rocket is increasingly dominating the total thrust. In fact Mach 6.0 is not used in the trajectory analysis, as the peak performance is less than that of a nuclear rocket. Swallowing air at this speed is a detriment; the drag imposed by the air intake is not compensated by the secondary combustion. At this point the inlet would be closed off and the engine operated as a nuclear rocket.

The variance between the thrust and air/fuel ratio tells another story. An examination of Figure 4 reveals that using progressively lower air/fuel ratios can increase thrust. The data also suggests that there is a change in slope of the constant Mach lines at the point of peak specific 
impulse. The right side of the peak efficiency, which are progressively leaner mixtures show a dramatic drop in thrust. This phenomenon is more pronounced as the Mach number increases. Conversely, the drop in thrust is less severe from fuel rich towards the peak efficiency point. Also notable is that the peak thrusts grow progressively higher with higher freestream Mach numbers.

\section{Mission Performance}

The best measure of the performance of a transatmospheric propulsion system is that of the delivered mass fraction to orbit. Determination of mass fractions typically requires knowledge of all of the forces acting on the vehicle for a range of freestream properties defined from ground level to orbit. The flight trajectory is then optimized to yield the best performance through tradeoffs of the produced thrust and experienced drag and gravity losses. Fortunately there are some simplifying assumptions that can be made to allow computation of a mass fraction to orbit without resorting to aerodynamic analysis of a vehicle and optimization of a flight trajectory. One assumption has already been made, that the vehicle in question will fly a trajectory similar to that illustrated by Billig. The ideal rocket equation combined with a mission-averaged specific impulse will determine the desired mass fraction to orbit.

Note that the NEAR concept only operates in the airbreathing modes of flight. Therefore different propulsion systems must be used in the non-airbreathing flight regimes. To increase efficiency these other propulsion systems would be related to the NBCC concept, using the same subsystems if possible. The NEAR mission analysis uses a nuclear rocket for thrust from Mach 6.0 to orbit. Its performance is assumed to be $800 \mathrm{sec}$., which is the minimum stated in ASPEN. The NBCC vehicle assumes that a ducted rocket is used from launch to Mach 2.0. The performance of this mode is assumed to be $500 \mathrm{sec}$. The nuclear reactor is not used in this mode in order to minimize civilian exposure to radiation. The $I_{s p}$ 's of the airbreathing mode will be determined by use of the SHX and NBCC codes.

The model first takes the specific impulse data as a function of freestream velocity. The model then calculates a mission averaged specific impulse by integrating the data using the trapezoidal rule. The mission-averaged specific impulse is then used in the ideal rocket equation to calculate the mass fraction. A delta- $\mathrm{V}$ of $30000 \mathrm{ft} / \mathrm{sec}$ is used in this equation, which corresponds to a $220 \mathrm{mi}$., 28.5 degree circular orbit and incorporates $4000 \mathrm{ft} / \mathrm{sec}$ for gravity and drag losses. The code then calculates the useful mass to orbit by subtracting the mass requirements for the engine. 
Figure 5 illustrates the relationship between specific impulse and freestream velocity for the concept. Note the straight lines, which represent the assumed performance of the nonairbreathing engine modes. The mission-averaged specific impulse for each concept is also illustrated. Note that the performance for the airbreathing mode in each concept is considerably higher than that of its non-airbreathing modes. The average performance using the QED rocket alone would be $2500 \mathrm{sec}$ (under the current assumptions). The calculated performance using the SHX in airbreathing mode yields a mission-averaged specific impulse of $3128 \mathrm{sec}$. The NEAR concept shows a similar increase in performance. The mission averaged performance using the ducted rocket and nuclear rocket would obviously be somewhere in the range of 500 and $800 \mathrm{sec}$ depending on the point of transition between the two engines. The calculated performance using the NEAR concept as well yields a mission specific impulse of $1277 \mathrm{sec}$. This suggests that use of the airbreathing concepts substantially increase mission performance.

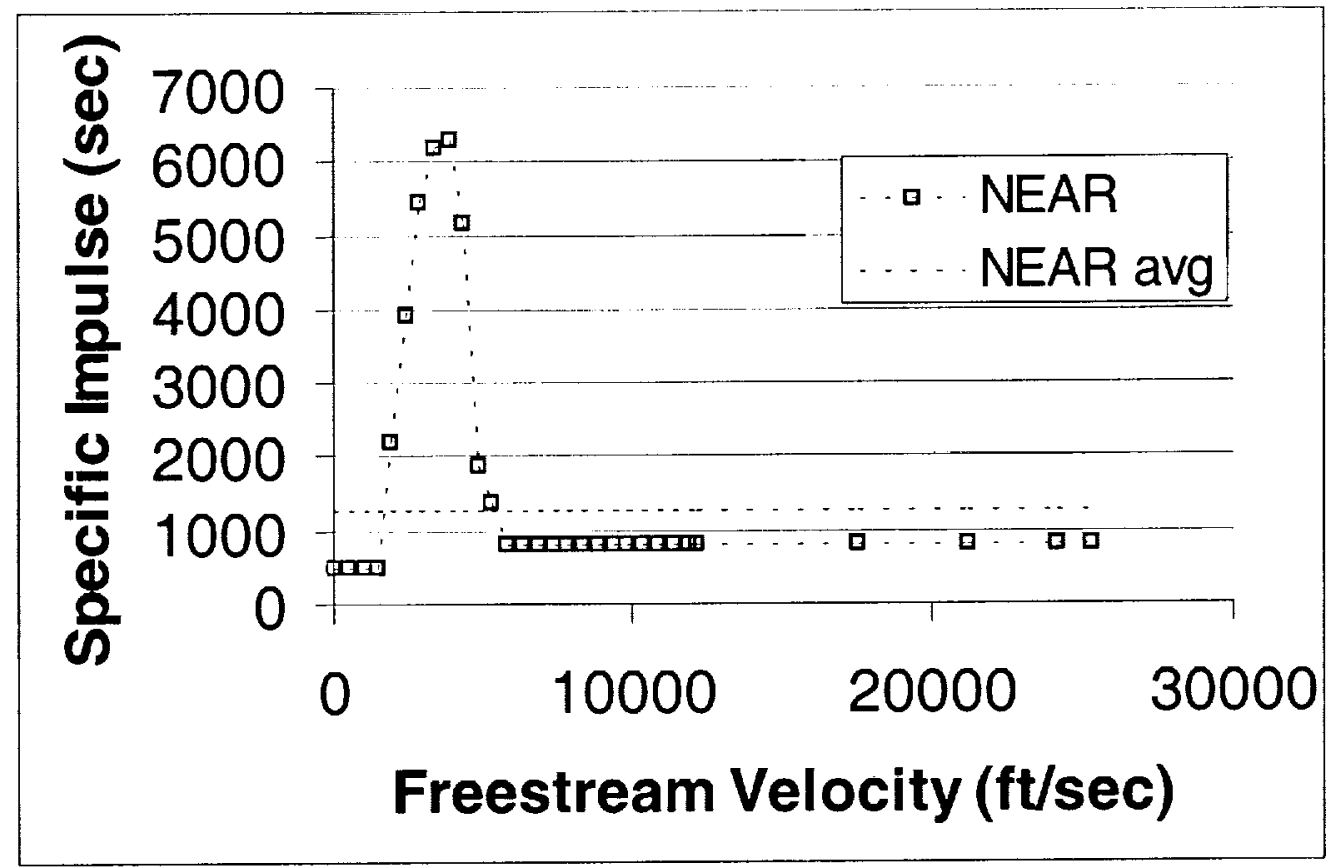

Figure 5 NEAR specific impulse vs. freestream velocity

The mass fraction to orbit was calculated using the ideal rocket equation. Table 3 shows the deliverable payload to orbit assuming a vehicle take off mass of $300,000 \mathrm{lbs}$, which is comparable to the mass of the shuttle (without the external tank or solid rocket boosters). The mass of the engine is a necessary part of the deliverable mass to orbit. Although the reactor mass may have some use in orbit for power production most of the mass is 'dead weight' once orbit is achieved. Therefore the mass of the engine is subtracted from the final mass to give a final 
available mass to orbit. This mass comprises the entire vehicle except the propellant burned in flight and the engine. Thus this is the mass available to designers for the vehicle structure, propellant tanks, ullage mass, avionics, crew facilities and payload. The NEAR mass available must also accommodate the thrust chamber, propellant pumps and coolant lines as the NEAR engine mass is comprised entirely of the fission reactor and shielding. These fractions to orbit are considerably higher than can be achieved by any existing propulsion system.

Table 3 Mission performance of NEAR concept

\begin{tabular}{ccccc}
\hline Concept & $\mathrm{M}_{\mathrm{f}} / \mathrm{M}_{\mathrm{o}}$ & $\mathrm{M}_{\mathrm{f}}$ & $\mathrm{M}_{\text {engine }}$ & $\mathrm{M}_{\text {avail }}$ \\
\hline NEAR & 0.482 & 144,560 & 8,735 & 135,825 \\
\hline
\end{tabular}

The only question that remains to be addressed is the capability of these propulsion concepts to fly the assumed trajectory. The issue is whether the concepts, as analyzed can produce the thrust necessary to accelerate along the trajectory profile. Figure 6 illustrates the thrust produced by the concept vs. the freestream Mach number. Immediately obvious is that the NEAR engine tends to produce more thrust at higher Mach numbers. The NEAR engine can increasingly rely on the nuclear rocket while still reaping the advantages of the secondary combustion. Lift generates a large portion of the vertical velocity for airbreathing vehicles. Therefore the engine accelerates the vehicle without having to counteract the gravitational force. The thrusts indicated in Figure 4 will accelerate a vehicle of 300,000 lb take off weight at a fraction to several g's. While such acceleration would not be sufficient to fly a rocket trajectory the thrusts indicated for the concept are likely to be sufficient to meet the trajectory requirements.

\section{Environmental Concerns}

One concern with nuclear engines is the effect such devices can have on the environment. A full addressing of such concerns was considered future work for this contract; however, some work was completed as part of this study. The NEAR reactor will definitely generate high levels of gamma and neutron radiation that will have to be attenuated. The NEAR model incorporates a standard shield configuration to address this issue. A more complete shielding model and calculation of impacts to the environment are left for a future study.

One other environmental concern for launch vehicles is the composition of exhaust gases. Launch vehicles can eject chemicals that will adversely interact with the atmosphere. 
Predictions were made of the chemical composition of the exhaust gases for the concept. The chemical composition of the NEAR engine exhaust is shown in Figure 7.

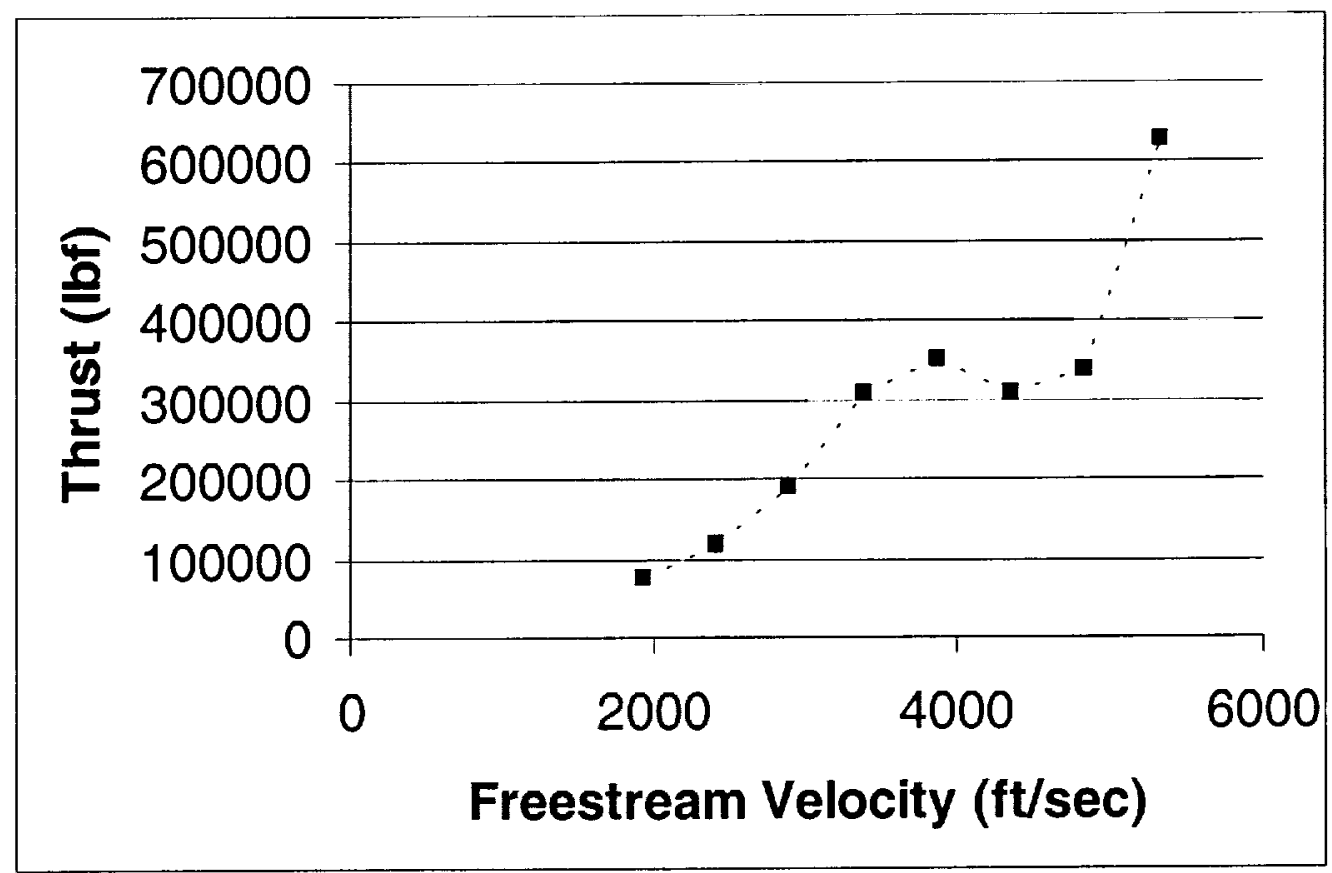

Figure 6 NEAR Thrust vs. freestream velocity

Trace amounts of ammonia $\left(\mathrm{NH}_{3}\right)$, methane $\left(\mathrm{CH}_{4}\right)$, nitrous oxides $\left(\mathrm{NO}_{\mathrm{x}}\right)$, and radicals that were neglected on this diagram for purposes of clarity. The largest amount of trace gases experienced was $0.22 \%$ of $\mathrm{NH}_{3}$ at Mach 5.5. Those gases, while harmful to the environment, are of such low quantities that their overall impact can be assumed to be minimal. The composition of the major gases left is very benign. Note that the hydrogen fraction increases sharply at the higher Mach numbers. This is indicative of the increased dependence on the nuclear rocket at higher speeds. One point worth mentioning is that the nuclear model is not currently able to calculate the amount of neutrons absorbed by the fuel. Thus, measurement of the isotopes of the above compounds is not possible at this time.

\section{Conclusions and Future Work}

The NEAR concept was developed using ISSI's EPSURBCC code as a template. All coding was done in Digital ${ }^{\Theta}$ Fortran PowerStation. Calculations were performed at strategically located stations through the flowfield. A model of a NERVA reactor design was incorporated. A mixing and combustion model was integrated into the code. Finally the code was adapted to take inputs from the HyFIM compression forebody model. 


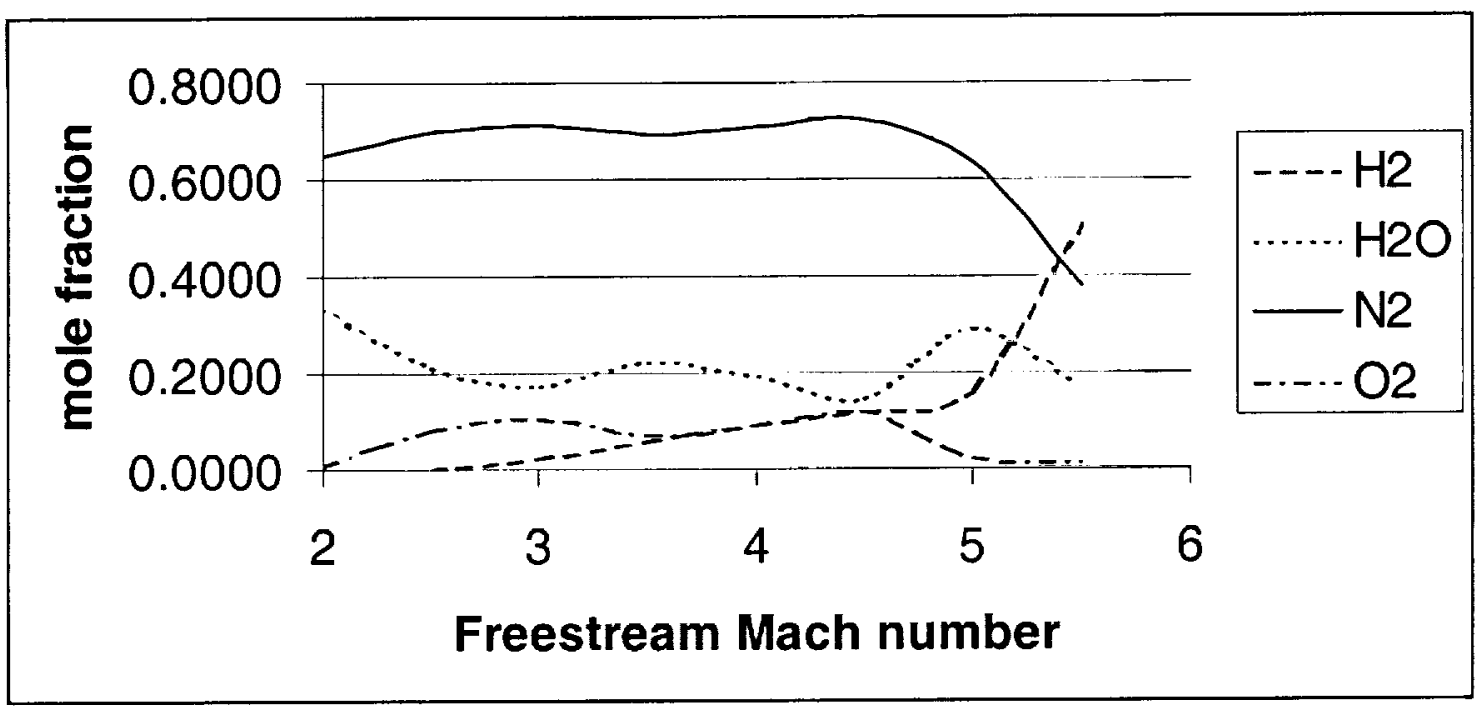

Figure 7 Composition of exhaust vs. freestream Mach number for NEAR concept

\section{NEAR Performance Characteristics}

The NEAR rocket was simulated using a series of air/fuel ratios for each Mach number tested along the trajectory. The selected air/fuel ratios were designed to capture the peak performance for the given Mach number. The peak performance was generally found at lean air/fuel ratios. The given engine geometry allows for only a certain level of expansion. Therefore peak performance is driven to lean ratios due to the limitation of the amount of thermal energy that the engine configuration can efficiently expand. Peak performance increases from Mach 2.0 to Mach 4.0 and then drops dramatically. Performance relies increasingly on the nuclear rocket as opposed to secondary combustion with the entrained air. Peak thrust generally increases with increasing Mach number. Thus there will be a tradeoff at higher Mach numbers between needed thrust and desired specific impulse.

\section{Future Work}

There are several areas in the NEAR analysis where a higher level of fidelity is desired. The air/fuel ratio has a strong impact on the engine performance. The ratio corresponding to peak performance is strongly affected by the engine geometry. A better understanding of these relationships could be realized through a parametric study varying engine geometry and air/fuel ratio. As mentioned above the NERVA reactor may not be the optimum reactor for a transatmospheric mission. Future work should address conversion to a particle bed reactor for 
increased performance. The NEAR code uses a 'rule of thumb' to address the possibility of backflow of the propellant exhaust (i.e. towards the engine air intake). A determination of backflow based on a more fundamental calculation would allow simulations eliminated by the 'rule of thumb'. Finally the NEAR code could be improved to allow for supersonic airstream flow. Such an improvement would make possible calculation of NEAR performance at higher freestream Mach numbers. 


\section{REFERENCES}

'Robbins, W. H. and Finger, H. B., "An Historical Perspective of the NERVA Nuclear Rocket Engine Technology Program”, AIAA Paper 91-3451, September 4-6, 1991.

${ }^{2}$ Angelo, Jr., J. A., Space Nuclear Power, Orbit Book Company, Inc., Malabar, FL, 1985.

${ }^{3}$ Lawrence, T. J., Witter, J. K., Humble, R. W., "Nuclear Rocket Propulsion Systems", Space Propulsion Analysis and Design, The McGraw-Hill Companies, New York, 1995.

${ }^{4}$ Dimotakis, P. E., "Turbulent Free Shear Layer Mixing and Combustion", High-Speed Flight Propulsion Systems, American Institute of Aeronautics and Astronautics, Inc., Washington, DC, 1991. 